A puntes del CENES

ISSN 0120-3053

Volumen 30 - №. 52

Segundo Semestre 2011

Págs. 183 - 202

\title{
Medición de la orientación al mercado de las empresas del sector artesanal en Boyacá y su incidencia en los resultados empresariales
}

\author{
Measuring of market orientation of \\ handicraft companies in boyacá and its \\ impact on business results
}

Sonia Yajqueline M ateus Riveros*

Edison Jair Duque Oliva**

F echa de recepción: 28 de marzo de 2011

Fecha de aprobación: 30 mayo de 2011

* Profesional en Mercadotecnia. Especialista en Alta Gerencia en Mercadotecnia Universidad Pedagógica y Tecnológica de Colombia - UPTC. Magíster en Administración. Convenio UNAL -UPTC. Gerente Banco WWB (Women's World Banking) Oficina Duitama. Docente cátedra UPTC. FESAD-Unipamplona. Correo electrónico: sonita262@gmail.com

** Administrador de empresas. Msc. en Marketing. Profesor asociado, Universidad Nacional de Colombia, Bogotá. Correo electrónico: ejduqueo@bt.unal.edu.co 
Medición de la orientación al mercado de las empresas del sector artesanal ...

Sonia Yajqueline M ateus Riveros - Edison J air Duque 0 liva

\section{Resumen}

El trabajo recoge una propuesta metodológica, a partir de una amplia revisión conceptual sobre la orientación del mercado, para estudiar la existencia de un comportamiento orientado al mercado en el sector de las artesanías en Boyacá, y la relación entre la orientación y los resultados. Con este propósito se analizan las concepciones propuestas por Narver y Slater (1990) y por Jaworsky y Kohli, (1993), se adopta la escala de medida de orientación al mercado M KTOR, de Narver y Slater, (1990), la cual indica que la OM es unidimensional, y está compuesta por la orientación al cliente, la orientación a la competencia e integración y la coordinación de funciones.

Palabras clave: orientación al mercado, sector de artesanías, rendimientos empresariales, análisis de escalas.

Clasificacion Jel: M 14, G14, M 21, M 31,

\section{Abstract}

The paper includes a methodological proposal, from a broad conceptual review ofmarket orientation, to study the existence of a market oriented behavior in the crafts sector in Boyacá, and the relationship between orientation and performance, to dowe study the concepts of market orientation proposed by Narver and Slater (1990) and Jaworsky and Kohli (1993), the adopted measurement scale MKTOR market orientation of Narver and Slater (1990), indicating that the OM is one dimensional, consisting of three components: customer orientation, competition orientation and integration and coordination of functions.

Keywords: Orientation to the market, Crafts Sector, business performance.

Jel Classification: M 14, G14, M 21, M 31 


\section{Introduccion}

En épocas anteriores, el principal fin de las empresas lo constituía el lucro, entendido como el beneficio buscado, producto de una actividad comercial y económica; en otras palabras, se perseguía crear valor para los accionistas. Pero los tiempos han cambiado, el avance de la tecnología, la globalización de los mercados, los movimientos ecologistas y ambientales, así como los nuevos hábitos de consumo de los clientes, ejercen una importante presión en las empresas para que consideren e incentiven la orientación al mercado como parte importante de la cultura empresarial. Este artículo busca exponer lo que representa la orientación al mercado, comprender su importancia del mismo y analizar la existencia de una relación entre las empresas de Boyacá del sector de artesanías dedicadas a los tejidos y la cerámica, que siguen una orientación al mercado, y los resultados empresariales que obtienen.

Con este objetivo se revisaron 68 referencias bibliográficas, principalmente artículos publicados en revistas académicas en relación con la orientación al mercado, a partir del año 1990 hasta la actualidad. El estudio de la literatura aquí presentada se enfoca en los aportes teóricos realizados principalmente en las últimas dos décadas, sin ignorar aportes clásicos necesarios para contextualizar la discusión académica contemporánea sobre el tema. Además, la revisión de la literatura se hace primordialmente sobre trabajos divulgados en publicaciones académicas. El enfoque abordado es netamente conceptual y pretende clarificar conceptos e incentivar la investigación futura en relación con la orientación al mercado. 
El sector artesanal en Colombia y en especial en Boyacá se ha caracterizado por tener una identidad propia, ubicado en una dimensión económica distinta de la industria, por su alto contenido de mano de obra y porque incorpora elementos históricos, culturales, estéticos y artísticos; por esto adquiere la categoría de obra de arte.

Por tal razón al artesano, debe considerársele como un patrimonio vivo, ya que en muchos lugares de Colombia, ha dinamizado su actividad productiva, con los recursos que le brinda la naturaleza, su creatividad y su contexto, pero muchas veces con ausencia de capacitación que facilite y articule su cotidianidad con las exigencias de las nuevas tendencias artesanales y los nuevos mercados, tanto para los artesanos como para sus artesanías.

Boyacá en las dos últimas décadas ha logrado ubicarse como una de las regiones colombianas con mayor potencial artesanal, gracias a la variedad de técnicas y materias primas empleadas, tal es el caso de la alfarería y los tejidos. La mano de obra de los artesanos boyacenses ha transcendido las fronteras nacionales y ha permitido que se facilite la comercialización directa de la artesanía boyacense. De acuerdo con Artesanías de Colombia, el mapa artesanal de este país muestra bien definidas las zonas productoras. Al sur, el departamento de Nariño es el núcleo principal, con un $14 \%$ de los artesanos del país. En el centro, Boyacá y Tolima representan respectivamente el $8 \%$ y el $5 \%$ del total nacional. El resto de la producción se ubica en los departamentos de la Costa Atlántica; Sucre con el $10 \%$, Córdoba con el $9 \%$, Cesar con el $7 \%$ y Atlántico con el $16 \%$.

El avance de la tecnología, la globalización de los mercados, los movimientos ecologistas y ambientales, los nuevos hábitos de consumo de los consumidores, y las disposiciones gubernamentales, ejercen una importante presión en las empresas. Estas acciones plantean la necesidad de adoptar en el sector artesanal del departamento de Boyacá, nuevos modelos de gestión que permitan la evolución de las empresas hacia una nueva cultura empresarial. Boyacá tiene varios núcleos artesanales reconocidos en el país por la originalidad y la calidad de su trabajo, como son la producción artesanal alfarera del municipio de Ráquira y los tejidos de Nobsa. Campos que se determinaron por ser los de mayor auge en el departamento.

No se trata solo de considerar la satisfacción de las necesidades del consumidor representado específicamente por el turista colombiano, como el eje orientador de la actividad empresarial, se trata entonces de adoptar una filosofía que vaya más allá de la simple satisfacción, una filosofía que involucre a toda la organización hacia una orientación al mercado que comprenda tanto factores internos (cliente interno) como externos (el mercado, clientes, competidores, tecnología, legislación) a fin de garantizar la satisfacción del cliente, por encima de sus propias expectativas. 
Se hace referencia a la orientación al mercado, la cual se ha convertido en un tema obligado para las empresas, por cuanto esta filosofía contribuye no solo a la satisfacción del cliente sino que tiene a favor la construcción de valores agregados que superen precisamente las expectativas de los clientes.

La literatura especializada en el tema sostiene que las empresas que adoptan el concepto de marketing y se orientan al mercado, obtienen mejores resultados empresariales (Flavian, \& Lozano, 1999, p. 1466-1471), tal como se evidencia en los desarrollos conceptuales y en las propuestas de escalas de medición (Jaworski \& Kohli, 1993, p. 53-70, 1996; Kohli y Jaworski, 1990: 1-18; Narver \& Slater, 1990, p. 20-35; Slater \& Narver, 1994, p. 46-55; 1995, P. 63-74; 1998, p. 1001-1006).

Siendo consecuentes con esta tesis, nuestro trabajo se centra en el análisis de la orientación al mercado y sus efectos en los resultados empresariales, en la empresa del sector de la artesanía en el departamento de Boyacá. La elección del sector responde a dos criterios:

En primer lugar, porque el sector se caracteriza por un enfoque hacia la venta, y por estar escasamente vinculado con la concepción del marketing. En segundo lugar, dada la importancia que ha adquirido el sector de artesanías para el departamento, tal como se refleja en los documentos Agenda interna para la productividad y la competitividad documento regional Boyacá, y Plan regional de competitividad de Boyacá 2008- 2032.

La orientación al mercado de una empresa es la consecuencia de la aplicación del marketing, por ello planteamos la necesidad de que el sector de las artesanías adopte la orientación al mercado como modelo de gestión con el objetivo de mejorar sus resultados. En consecuencia, el objetivo fundamental de este trabajo es estudiar la orientación al mercado de las empresas de artesanías en el departamento de Boyacá, planteando y validando una escala de medición que permita expresar el grado de la orientación al mercado y su incidencia en los resultados empresariales.

Este documento está dividido en seis secciones: en la primera se presentan algunas generalidades del sector de las artesanías en el departamento de Boyacá y del concepto de orientación al mercado. La segunda muestra la revisión literaria, destacándose dos corrientes de pensamiento: la orientación al mercado como filosofía y la orientación al mercado como comportamiento, y la relación existente entre la orientación al mercado y los resultados de la empresa. En la tercera se describe el objetivo de la investigación, el cual es comprobar de qué manera el grado de orientación al mercado influye positivamente en el rendimiento empresarial de las empresas de artesanías en el departamento de Boyacá. En la cuarta, se exponen las características de la escala de medida MKTOR. En la quinta se presenta el diseño metodológico que 
se desarrollará en la investigación, el cual toma como base el modelo propuesto por Narver y Slater (1990, p. 20-35), Slater y Narver (1994, p. 46-55): modelo de orientación al mercado como coordinación interfuncional de la información del mercado. Finalmente se presentan las conclusiones que ha arrojado el estudio.

\section{Revisión literaria}

Muchos de los estudios guardan una estrecha relación en cuanto tratar de generar un concepto que defina de alguna manera "la orientación al mercado" (Desphandé et al., 1993: 23-27; Narver \& Slater 1990, 20-35 Slater \& Narver 1994, 46-55, Cadogan \& Diaman-topoulus, 1995, 41-60; Jaworski \& Kohli, 1993, 53 70; Kohli et al., 1993, 467-477; Cadogan et al., 2001, 261-282, 2002, 615-626, 2003, 493- 513, 2006, 634-647; Álvarez et al., 2005, p. 161-202) Se destaca la interacción de factores tanto externos -clientes, competencia-, como internos -cliente interno-, a la organización, los cuales se constituyen en los elementos dinámicos en la orientación al mercado de las empresas.

Sin embargo se acentúan dos corrientes de pensamiento sumamente importantes, la orientación al mercado como filosofía (Narver \& Slater, 1990, p. 20-35) y la orientación al mercado como comportamiento (Kolhi \& Jaworski, 1990, p. 1-18).

Naver y Slater, (1990, p. 20-35), son los primeros en establecer una escala de medición de la orientación al mercado desde el punto de vista actitudinal, poniendo el acento en el carácter de competitividad y en la generación de una ventaja competitiva sostenible. Los autores afirman que el deseo de crear un valor superior para los consumidores y lograr conducir el negocio con una ventaja competitiva sostenible se basa en crear y mantener una filosofía que producirá las conductas precisas.

Y es precisamente la orientación al mercado, la cultura de organización que más efectiva y eficientemente crea los comportamientos necesarios para la creación de ese valor superior, permitiendo además continuar con una rentabilidad superior para el negocio. Los autores configuran también su propio modelo de orientación al mercado. Al igual que Kohli y Jaworsky (1990, p. 118), parten de una revisión literaria, con el propósito de formular una definición operativa y su posterior validación que sirva para medir el grado de orientación al mercado y su relación con los resultados de la empresa.

De este análisis concluyen que la orientación al mercado se compone de tres aspectos: orientación al cliente, orientación a la competencia, coordinación interfuncional, así como dos criterios de decisión: enfoque a largo plazo y rentabilidad, que pueden ser medidos con un alto grado de fidelidad.

La revisión de la literatura lleva a los autores a concluir que la orientación al mercado posee las siguientes caracterís- 
ticas: orientación al cliente, integración y coordinación de las funciones de la empresa, y orientación al beneficio.

En el trabajo de Kohli y Jawoski (1990, p. 1-18), se establece que una organización orientada al mercado será aquella en la que estos tres pilares se manifiestan de forma operativa; por tanto, es imprescindible trasladar la filosofía a la práctica a través del desarrollo de un conjunto de actuaciones para llegar a una efectiva orientación al mercado. Para conseguir la aplicación del marketing y que se concrete en una auténtica orientación al mercado, hace falta incorporarle al concepto un componente más operativo y no tan ideológico.

Con este propósito de estructurar el componente operativo, diseñan un modelo que explica la orientación al mercado, basado en las consideraciones previas, es decir, la orientación al cliente, la integración de las funciones de la empresa y la orientación al beneficio. Sin embargo, el modelo presenta factores adicionales; se trata de los antecedentes o condicionantes para la orientación al mercado, de los factores que pueden moderar el efecto de la misma, así como los resultados que esta genera, más conocidos como antecedentes, modificadores y consecuencias.

Al hacer un análisis detallado de los planteamientos de Kohli y Jaworski, (1990, p. 1-18 y de Narver y Slater (1990, p. 2035 ), se pone de manifiesto la existencia de bastantes puntos en común entre ambos enfoques, tal y como posteriormente admiten Slater y Narver (1994, p. 46-55). Comparando estos componentes con los aportados por Kohli y Jaworski (1990, p. 1-18), encontramos grandes similitudes. Los tres componentes de orientación al mercado mencionados por Naver y Slater (1994, p. 20-35) comprenden actividades de adquisición de información de mercado, de su diseminación y coordinación en la creación de valor para el cliente, que resultan formar parte de los pilares de la orientación al mercado, según Kohli y Jaworski (1990, p. 1-18).

Así, la orientación al mercado y la orientación a la competencia incluyen actividades referentes a la adquisición de información sobre compradores y competidores en el mercado objetivo, y de su difusión por toda la empresa. Por otro lado, el tercer componente de coordinación interfuncional comprende los esfuerzos coordinados que van más allá del departamento de marketing y que crean un valor superior para el cliente.

Continuando en la revisión literaria, una de las hipótesis de mayor estudio es la que tiene que ver con el tipo de relación existente entre la orientación al mercado y los resultados empresariales. La asociación entre orientación al mercado y resultados empresariales, aparece reflejada en los estudios de Kohli y Jaworski (1990, p. 1-18). Estos autores establecen en su análisis, que esta relación plantea una serie de variables que actúan como moderadoras de la relación; entre estas variables se 
encuentra el nivel de turbulencia, la intensidad del mercado y la tecnología, la tasa de crecimiento del mercado, etc. (Bigne, 2005, p. 33-58; Llonch \& López, 2004, p. 65-88; Lee, 2005, p. 345-48)

Desde la perspectiva de Naver y Slater (1994, p. 20-35), también se argumenta la existencia de una relación positiva entre los dos aspectos. Los autores consideran que la rentabilidad de la empresa depende de tres factores: a) características del mercado, b) características propias del negocio, c) grado de orientación al mercado de la empresa (orientación al consumidor, orientación a la competencia, coordinación interfuncional). Ellos encontraron evidencia de una fuerte relación entre orientación al mercado y resultados, una vez llevados a cabo los ajustes correspondientes por los efectos moderadores en el ámbito de mercado y en el ámbito de empresa.

Recientemente se atribuye a la orientación al mercado un papel determinante a la hora de explicar el resultado de las empresas (Cadogan et al., 2001, p. 261282, 2002, p. 615-626, 2003, p. 493- 513, 2006, p. 634-647; Álvarez et al., 2005, p. 161-202), porque las actividades relacionadas con la orientación al mercado proporcionan información y conocimiento sobre los clientes, los competidores y el entorno (Slater \& Narver, 1995 , p. 63-74). De igual manera se ha considerado el rendimiento empresarial como un concepto multidimensional que integra medidas de eficacia, eficiencia y adaptabilidad.
No obstante, también hay que reseñar otro de los aspectos que se han considerado, y es el que tiene que ver con el desempeño del negocio entendido como el resultado empresarial. El desempeño es un concepto que conduce a englobar diversos factores que pueden ser medidos y cuantificados; se refiere de manera conceptual a la realización de responsabilidades gerenciales con atributos de calidad, por lo que puede decirse que se relaciona con rentabilidad, eficiencia, oportunidad, congruencia, resultados, efectividad y factibilidad en la toma de decisiones (Hernández, 2002).

En esta misma línea, O’Regan, Sims y Ghobadian (2005) consideran que el desempeño es la habilidad de un objeto para reproducir resultados en una dimensión considerada a priori en relación con los objetivos. De igual manera, Martínez (2004) enfatiza que la organización con una filosofía orientada al mercado beneficiará a la organización en la obtención de mejores resultados en su desempeño. En este mismo sentido, Nahm (2005, p. 45-54) menciona que el desempeño incluye crecimiento en ventas, retorno sobre la inversión, ganancias del mercado y posición competitiva en general.

Por su parte Hult, Hurley y Knight (2004, p. 42-54) definen el desempeño del negocio como el logro de los objetivos organizacionales relacionados con la rentabilidad y crecimiento en ventas y posición en el mercado, así como el cumplimiento de los objetivos 
estratégicos generales del negocio, en tanto que para Hooley, et al., (2005, p. 18-27) la medición del desempeño es necesario agruparla en desempeño del mercado, del cliente y financiero.

El desempeño del mercado está compuesto por el crecimiento en ventas y la posición del negocio en el mercado. El desempeño financiero, por los niveles logrados de los ingresos en comparación con los competidores, aumento en los márgenes de ganancias y la reinversión. El desempeño del cliente se refiere a la lealtad del mismo y a los niveles de satisfacción del cliente en relación con los competidores.

A lo largo de la evaluación de la literatura, se nota la ausencia de un consenso sobre qué y cómo se deben medir los resultados de la empresa. Para algunos, el principal resultado es el beneficio, para otros, la rentabilidad (Venkatraman \& Vasudevan, 1986, 801-814), para otros, el margen sobre las ventas (Buzzel, Bradley \& Galey, 1975, p. 97-106) y para otros la participación en el mercado (Despande \& Farley, 1990, p. 23-37). Esta diversidad de acepciones confirma las conclusiones de las investigaciones de Bourgeois (1980, p. 27-248) y de Galbraith y Schendel (1983, p. 153-173), en el sentido de que los resultados de la empresa no son un concepto único, sino que está compuesto por múltiples subobjetivos. Según Anderson (1988, p. 15-26), el principal objetivo de la implementación del concepto de marketing es la maximización del beneficio, de ahí puede deducirse que el principal criterio para determinar el resultado es el beneficio Chaffe, 1988, p. 157-176).

Sin embargo, en la literatura también se encuentran sistemas muy variados para cuantificar la variable resultados. Esto es tan cierto que, como resultados empresariales se han contemplado tanto los resultados financieros (ROI, ROA, beneficios), como operativos (ventas, cuotas de mercado) y los relativos a la eficacia del marketing (nuevos productos, distribución alcanzada, satisfacción del cliente).

Los indicadores allí presentados tienen que ver con formas comunes de tipo financiero, como el rendimiento sobre los activos ROA, rendimientos sobre la inversión ROI, de tipo operativo, como las cuotas de mercado, y el crecimiento de las ventas. En general, los resultados de estas investigaciones demuestran la existencia de una relación positiva entre OM y los resultados empresariales.

Así las cosas, el rendimiento es un fenómeno con múltiple facetas, siendo necesario el uso de un conjunto de medidas que lo evalúen desde distintos ángulos, para minimizar así las limitaciones que puedan tener individualmente cada una de estas facetas (Evangelista, 1994).

Para efectos de la investigación, se toman los indicadores financieros de rentabilidad, plasmados en la rentabilidad sobre la inversión, margen de ventas. 
Consideramos que es necesario medir el éxito empresarial, representado en la rentabilidad, para lo cual se requieren utilizar los indicadores financieros y operativos, con el fin de no limitar la interpretación de los resultados de la investigación.

De otro lado, la literatura ha demostrado que la orientación al mercado establece relaciones positivas con las mismas, de hecho, una mayor comprensión acerca de los clientes y los competidores por parte de la empresa, proporciona una mejor toma de decisiones, más efectiva, que conduce a un mayor crecimiento de las ventas y por ende en el margen de ventas. Reicheeld dice que "cuando una empresa, de forma consistente oferta un valor superior y consigue la lealtad de sus clientes, crecen la ventas, los ingresos y disminuyen los costos de servir a los clientes" (1993, p. 64-73).

Además, la orientación al mercado parece proveer una atención unificada de los esfuerzos de los actores de la organización, dando lugar, desde luego, a un desempeño superior, lo cual redunda de manera beneficiosa para el rendimiento de la organización.

En este apartado se ha analizado el concepto de la orientación del mercado desde dos corrientes de pensamiento muy influyentes, siendo estas coincidentes, en el sentido que la orientación al mercado proporciona más y mejores resultados en las empresas que la implementan.

\section{Objetivo del trabajo}

La orientación al mercado se ha convertido en un tema obligado para las empresas, por cuanto esta filosofía permite determinar la relación entre la $\mathrm{OM}$ y los resultados. Por ello, la literatura especializada en el tema afirma que las empresas con un alto grado de orientación al mercado obtienen más y mejores resultados, por cuanto tienen la disponibilidad de identificar y satisfacer las necesidades de sus clientes (Singh \& Ranchod, 2004, p. 137-154).

Siendo consecuentes con esta tesis, planteamos la necesidad de que las empresas del sector de artesanías adopten la orientación al mercado como modelo de gestión empresarial para obtener mejores resultados. Nuestro objetivo es precisamente evaluar la relación existente entre la orientación al mercado y los resultados globales de la empresa del sector de las artesanías en el departamento de Boyacá. El segundo objetivo consistirá en analizar la existencia de una relación positiva entre la orientación al mercado y los resultados empresariales con base en estudios previos que evidencian dicha relación.

\section{La escala de medida de orientacion al mercado}

Para la consecución de estos fines, se ha optado por aplicar la escala MKTOR, propuesta por Narver y Slater (1990, p. 46-55). El hecho de utilizar la escala, se debe a una doble motivación. En primer lugar, al convencimiento personal de que 
dicho criterio es uno de los principales y fundamentales para evaluar los resultados de la empresa. De otro lado, al hecho de que en la investigación formulada por Narver y Slater (1990) sobre los efectos de la orientación al mercado en los resultados de la empresa, se tomó como criterio de medición la rentabilidad, tratándose de la primera investigación de este tipo, por tanto creemos de interés adoptar este mismo discernimiento.

La escala de medición de la orientación al mercado MKTOR, debe ser entendida como una coordinación interfuncional de la información relacionada con el mercado. Principalmente basada en los primeros trabajos desarrollados por Narver y Slater (1990, p. 46-55) y Slater y Narver (1994, p. 20-35), esta línea de estudio propone que la orientación al mercado sea entendida como una cultura organizacional.

Esta cultura se fundamenta en la conjunción de tres componentes: orientación al consumidor, orientación a la competencia, coordinación interfuncional. Los dos primeros componentes incluyen actividades relacionadas con la adquisición de información y posterior diseminación de la misma dentro de la empresa, y el tercero, basado en la información del mercado, trata de crear mayor valor para los compradores.

De las consideraciones realizadas en el modelo de Narver y Slater (1990, p. 2035 ), dedujimos que cada uno de los tres componentes de la orientación al mercado tiene la misma importancia para el modelo, por lo tanto el grado de orientación al mercado dependerá igualmente de los valores que alcance la empresa en orientación al consumidor, orientación a la competencia coordinación interfuncional.

La escala recoge los tres componentes que los autores apuntan en su desarrollo teórico: 1) orientación al cliente, 2) orientación a la competencia y 3 ) coordinación interfuncional. Cada uno de estos componentes está formado por una serie de ítems que hacen un total de quince, los cuales deben ser valorados con una escala de Likert de 1 a 7 puntos. Dicha escala la aplicaron a 140 unidades estratégicas de negocio de una única corporación. Las valoraciones obtenidas permitieron constatar la validación y la fiabilidad de la misma.

La escala MKTOR ha gozado de una notable aceptación en la literatura con posterioridad al trabajo de Narver y Slater (1990). Otros estudiosos del tema han seguido la misma línea de pensamiento, incluidos los propios autores, que continuaron con el análisis de la orientación al mercado como filosofía, entendida como una cultura empresarial. Entre ellos se encuentran Slater y Narver (1994a, p. 22-28; 1994b, p. 230-232; 1995, p. 63-74), Day (1994, p. 37-52), Greenley (1995), Llonch (1993), Chan, Hung, Ngai y Ellis (1998, p. 119-150), Kim y Hwang (1992, p. 29-53), Chang y Chen (1998, p. 246-264), Narver, Slater y Tietje (1998, p. 233-236), Becker y Homburg (1999, p. 
17-41), Martin y Armario (2003, p. 3-81), Scarone y Delgado (2005, p. 31-48), Gayner y Padayani (2005), Samino y García (2007).

En las empresas de artesanías, la orientación al mercado como filosofía implica que todas las áreas de la organización adopten como manera de pensar y concebir las relaciones de intercambio centradas en satisfacer las necesidades de sus públicos objetivos mejor que los competidores. De esta manera se conseguirá crear una ventaja competitiva sostenible en el tiempo, que se traducirá en mejores resultados y en la supervivencia a largo plazo

\section{Metodología de la investigación}

Este estudio incluye una primera fase de investigación preliminar en donde se revisó la bibliografía relacionada con la temática para la adopción de la escala, como paso previo a la aplicación del instrumento. El cuestionario se dividió en tres partes: en la primera están los datos relativos a la empresa, en la segunda parte hay preguntas propias de la investigación (componentes de la orientación al mercado) y en la tercera hay aspectos propios de los resultados empresariales.

El instrumento se aplicó en una muestra piloto de empresas del sector, con el objetivo de detectar ambigüedades o interpretaciones incorrectas. Las empresas a las que se les aplicó este pretest no se incluyeron en la muestra final. Para la investigación se tomó como unidad poblacional, las empresas del sector de artesanías que se encuentran registradas en cámara de comercio. La muestra se seleccionó con base en el método aleatorio simple, intentando abarcar a los municipios en los que la artesanía es más representativa, y de acuerdo con una ruta que incluyera el mayor número de comunidades del departamento de Boyacá, especialmente en los municipios de Nobsa y Ráquira, obteniéndose así una muestra de 186.

Para el desarrollo de una escala específica que permitiera medir el grado de orientación al mercado de las empresas del sector de las artesanías del departamento, fue necesaria la adopción de la escala MKTOR de Narver y Slater (1990, p. 20-35), así, una vez adaptada la escala al contexto, se procedió a su evaluación.

Para realizar el procedimiento de evaluar una escala de medida, se seguirá el procedimiento propuesto por Churchill (1979, p. 64-72) y por Anderson y Gatignon (1986, p. 1-26), analizando la validez de contenido, la consistencia interna de la escala (fiabilidad), la validez de concepto o constructo. En primera instancia se analiza la validez de la misma, valga decir, el grado en que el instrumento de medida que se utiliza mide realmente el concepto que se quiere medir.

La validez de contenido busca comprobar si los indicadores empleados para medir 
un fenómeno determinado cubren todos sus aspectos o dimensiones. Esta validación es eminentemente teórica y subjetiva, se lleva a cabo por medio de una profunda revisión de la literatura pertinente (Flavián \& Lozano, 2001, p. 1466-1471) y de una posterior adaptación y depuración de los ítems. Hecho que se ve reflejado en la investigación, tal como lo manifiesta Lado (1999, p. 571-590). Los pretest del cuestionario y la opinión de los distintos autores indican que el contenido de la escala estaá adecuadamente representado.

En resumen, con el análisis de contenido se busca garantizar que el instrumento evaluado contenga los aspectos que lo caracterizan. Hair, et, al. (1999, p. 105) lo definen como la evaluación de las correspondencias de las variables incluidas en la escala con su definición conceptual y que sirve para valorar de forma subjetiva la correspondencia entre los ítems individuales y el concepto mediante la evaluación de expertos.

Un aspecto clave del estudio es la validez interna, puesto que esta exige que la información suministrada provenga de fuentes adecuadas. Así pues, se selecciona como única fuente de recolección de información al responsable de la organización, conocedor tanto quien es del funcionamiento interno como externo de la misma y del sector de actividad, de forma que su conocimiento sea tan profundo como general. La elección de este enfoque se ha llevado a cabo con anterioridad en otros estudios, en los que se ha escogido un único encuestado para así fortalecer la validez de contenido de las escalas de medida empleadas. (Narver \& Slater, 1990, p. 20-35; Avlonitis \& Gounaris, 1997, p. 385-402; Bennett, 1998, p. 243; Álvarez \& González, 2000, p. 7-41).

La segunda etapa es el análisis de fiabilidad. Una escala de medida se considera fiable cuando los indicadores que la estructuran se encuentran altamente correlacionados (Nunally, 1979; Churchill, 1979, p. 64-72). La fiabilidad se refiere al grado en que sus mediciones están libres de errores aleatorios, de tal forma que proporcione resultados empíricos consistentes y estables.

De esta forma para analizar la fiabilidad de la escala de orientación al mercado se utiliza el coeficiente Alpha de Cronbach, que trata de comprobar el grado en que los indicadores o ítems de la escala miden el mismo concepto. Su valor esta entre 0 y 1. La alta correlación entre los ítems de la escala (valores a partir de 0,7 ) puede considerarse que la escala goza de consistencia interna (Nunally, 1979).

Una vez realizado el análisis de fiabilidad, es necesario estudiar la validez de concepto. Se refiere a la naturaleza del constructo que se pretende medir, y busca evaluar si de acuerdo con la teoría, existe una correspondencia entre una construcción conceptual y la escala que se ha propuesto para su medición. En otras palabras se trata 
de determinar qué está realmente midiendo el instrumento de medida (Luque, 1997, p. 254).

La validez de concepto se comprueba en dos aspectos: validez convergente, establece que si los indicadores de un constructo miden lo mismos, deben estar fuertemente correlacionados entre sí de forma significativa. Cabe anotar que la validez convergente viene influida positivamente por la fiabilidad, de tal suerte que a mayor fiabilidad mayor validez convergente (Peter \& Churchill, 1986, p. 64-72). Y la validez discriminante hace referencia al débil grado de relación que existe entre indicadores que miden conceptos diferentes, es decir, un concepto debe ser muy distinto de otro como para que esté justificada su existencia (Lehmann et al., 1999, p. 255).

\section{Conclusiones}

Revisados los distintos matices conceptuales encontrados en la literatura especializada, la orientación al mercado podría definirse como un enfoque de gestión en las empresas que adoptan el concepto de marketing. Es una característica de la cultura empresarial que nace desde el marketing, en la que el mercado, los competidores y los clientes son el eje fundamental de las operaciones de la empresa, con el constante propósito de satisfacer las necesidades de los clientes de forma continuada.

El estudio permite establecer la necesidad de implementar modelos de gestión en las empresas del sector de las artesanías en el departamento de Boyacá, como respuesta a las nuevas condiciones del mercado (Departamento Nacional de Planeación, 2007), producto del nuevo marco competitivo mundial, el cual plantea nuevas situaciones y oportunidades. Para responder a estas nuevas concepciones, las empresas del sector de las artesanías deben estar en condiciones de detectar amenazas del entorno, pero, sobre todo, de detectar las necesidades presentes y futura, de sus clientes, con el propósito de satisfacerlas. Para ello las empresas deben adoptar una orientación al mercado como modelo de gestión, de tal manera que les permita obtener ventajas comparativas y así ser más competitivas.

Respecto a la medición del grado de orientación en que una organización está orientada al mercado, el objetivo fundamental del presente trabajo se ha centrado en torno a la aplicación y validación de la escala de medida de la orientación al mercado MKTOR, propuesta por Narver y Slater (1990, p. 20-35) que permite medir el grado de orientación al mercado seguido por las empresas del sector artesanal del departamento de Boyacá.

Sobre la base de la aplicación y evaluación de la escala de medida de orientación al mercado MKTOR, la cual determina la existencia de una relación positiva entre la orientación al mercado y los resultados empresariales de las empresas del sector, en el sentido que a mayor orientación al mercado mejores resultados. 
De cualquier modo, la mayoría de las investigaciones existentes sobre la orientación al mercado y los efectos sobre los resultados y de la escala de medidas utilizadas en distintos sectores, se ha concluido la existencia de una relación positiva entre la orientación al mercado y los resultados.

A la fecha son poco los trabajos que se han dedicado a analizar la orientación al mercado en empresas del sector artesanal y de otros sectores del departamento, salvo estudios que tienen que ver con la historia del pueblo boyacense, (Ocampo, 1983), la estructura y la organización gremial de la producción, (Herrera, 1992), la artesanía y el diseño en Colombia, (Quiñones, 2003), las artesanías de Colombia, (Mandowky, 2004), o el estudio de la artesanía mexicana; (Mejía, 2003), los cuales están más centrados en el sentir de la producción de la artesanía. Estudios que se han realizado desde la antropología o desde la sociología, mas no desde el comportamiento como empresa de la organización artesanal. Por estas razones, con este estudio se pretende estimular la investigación en la aplicación de nuevos modelos de gestión que les permita a las empresas ser cada vez más competitivas.

\section{R eferencias bibliográficas}

1. Álvarez, L. Santos, M y Vásquez R.(2005). Escalas de medida del concepto de orientación al mercado: revisión crítica de su contenido y de sus prioridades psicométricas. Esic market. $\mathrm{N}^{\circ} .05$ enero abril, pp. 161-202.

2. Anderson, P. (1982). Marketink, strategic planning and the theory of the firm.Journal of Marketing, 46, 15-26.

3. Álvarez, L. I., Santos, M. L. \&Vázquez, R. (2003). La orientación al mercado cultural en el contexto de las organizaciones privadas no-lucrativa: propuesta y evaluación de una escala de medida.Revista europea de dirección y economía de la empresa, 12 (1),177-188.

4. Avlonitis, G, J. \&Gounaris, S.P. (1997). Marketing orientation company performance: industrial vs consumer goods companies. Industrial marketing management, 26. (5), 385-402

5. Baker, W. E. \&Sinkula, J.M. (1999).The synergistic effect of market orientation and learning orientation on organizational performance.Journal of the academy of marketing science,27, (4), 411-427.

6. Becker, J. y Homburg, C. (1999).Marketoriented management: a system- based perspective. Journal of market focused management, № 4, pp.17-41.

7. Bennet, R. (1998).Businness Associations and their potential contribution to the competitiveness of SMEs. Entrepreneurship and regional development, 10, 243.

8. Bigné, E. \&Blesa, A. (2002). Una 
concepción ecléctica de la orientación al mercado y su escala de medición.Revista española de investigación de marketing ESIC,6,(2), 33-58.

9. Bourgeois, L. J. III.(1980).Making your marketing strategy work.Harvard Business Review, 1, 27-248

10. Buzzel, R., Bradley, T. \&Galey, R. (Enerofebrero, 1975). Market share, a key to profitability.Harvard Business Review, 97106

11. Cadogan, J. W. \& Diamantopoulos, A. (1995).Narver and Slater, Kohli and Jaworski and the marketing orientation construct: integration and internationalization. Journal of strategic marketing, 3 (4), 41-60.

12. Cadogan, J. W., Cui, C. C., Morgan, R. E. \& Story, V. M. (2006). Factors facilitating and impeding the development of export market-oriented behavior: a study of Hong Kong manufacturing exporters.Industrial Marketing Management, 35,634-647.

13. Cadogan, J. W., Cui, C. C. \&Yeung Li, E. K. (2003). Export market-oriented behavior and export performance: the moderating roles of competitive intensity and technological turbulence.International Marketing Review, 20 (5), 493-513.

14. Cadogan, J. W., Diamantopoulos, A. \&Siguaw, J. A. (2002).Export marketoriented activities: their antecedents and performance consequences.Journal of International Business Studies, 33 (3), 615626.

15. Cadogan, J. W., Paul, N., Salminen, R. T., Puumalainen, K. \&Sundqvist, S. (2001). Key antecedents to export market-oriented behaviors: a cross-national empirical
examination.International Journal of Research in Marketing, 18 (3), 261-282.

16. Cambra, J. J. \& Fuster, A. (2005). Importancia de la medición del grado de orientación al mercado en el sector cooperativista como respuesta al nuevo marco competitivo. Investigaciones europeas de dirección y economía de la empresa, 11 (3), 67-83.

17. Caruana, A. (1999). An assessment of the dimensions and the stability of items in the markor scale.Marketing intelligence \& planning, 17(5), 248-253.

18. Chaffee, E. (1988)The impact of resource predictability and management strategy on performance.s.1.: John Wiley and Sons Ltd., 157-176.

19. Chan, Hung,Ngai\& Ellis.(1998).Market orientation and business performance: some evidence from Hong Kong. International marketing review, 15, (2),119-150.

20. Chang, T. Z. \& Chen, S. J. (1998).Market orientation, service quality and business profitability: a conceptual model and empirical evidence. The journal of services marketing, 12 (4),246-264.

21. Churchill, G. A.(1979).A paradigman developing better measures of marketing constructs.Journal of Marketing Research, $16(1), 64-73$.

22. Day, G. (Octubre, 1994). The capabilities of market-driven organizations.Journal of marketing, 58 (10), 37-52.

23. Day, G. \&Nedundadi, P. (Abril, 1994).Managerial representations of competitive positioning.Journal of marketing, 58 (4),31-44. 
24. Deshpande, U., Farley, J.U. \& Webster, F. E. (Enero, 1993). Corporate culture, customer orientation, and innovativeness in japans firms: a quadratic analysis", journal of marketing, 57 (1),23-27.

25. Deshpande, R., Farley, J. U. \& Webster, F. E. (2000).Triad lessons: generalizing results on high performance firms in five business-to-business markets.International journal of research in marketing, 17 (2),353-362.

26. Deshpande, R. \& Webster, F. E. (Enero, 1989). Organizational culture and marketing: defining the research agenda. Journal of marketing, 53 (1),3-15.

27. Ellis, P. D. (2006).Market orientation and performance: a meta-analysis and crossnational comparisons. Journal of management studies, 43 (5), 1089-1107.

28. Evangelista, F.U. (1994). Export performance and its determinants: some empirical evidence from Australian manufacturing firms. Advice International Marketing, Vol.6, pp.207-229.

29. Flavian, B.C. \& Lozano, F.J. (Mayo, 2001b).Market orientation in the Spanish education sector.En $30^{\text {th }}$ European Marketing Academy Conference (EMAC). (pp. 1466-1471).Conferencia llevada a cabo en Bergen, Noruega.

30. Galbarith, y Schendel. (1983). An empirical analysis of Strategys Types. Startegic Management Journal, Vol. 4, pp.153-173.

31. Gainer, B., yPadanyi, P. (2005). The relationship between market-oriented activities and market-oriented culture: Implications for the development of market orientation in nonprofit service organizations. Journal of Business
Research, 58(6), pp. 854-862.

32. Gounaris, S.P. y Avionitis, G.J. (1997). Company and marketing correlatos of marketing orientation development: an empirical investigation. Inglaterra: Proceedings of 26th EMAC Conference, 2oth - 23rd may. Warwick, vol. 1

33. Hair, J. F., Anderson, R.E., Tatham, R.L. \& Black,W.C. (1999). Análisis multivariante. ( $5^{\mathrm{a}}$. Ed.).Madrid: Prentice Hall Iberia.

34. Hernández, C. y Martín, C. (2000). Estudio de Mercado vs Despliegue Funcional de la Calidad: La Matriz QFD como superación de las limitaciones de los enfoques de Recursos-Capacidades y del Marketing: de Orientación al Mercado. X Jornada Hispano-Lusas de Gestión Científica, febrero, pp 113-120.

35. Herrera, N. (1992). Artesanía y organización social de su producción. Bogotá: Centro de Investigación y Documentación, Artesanías de Colombia.

36. Hooley, J.G., Cox, T., Breacs, J., Fonfara, K. \& Snob, B. (2000b).Market orientation in the transition economies of central Europe: test of the Narver y Slater market orientation scale. Journal of Business Research, 50 (3), 273-285.

37. Hunt, S. D. \& Morgan, R. M. (1996). The resource-advantage theory of competition: dynamics, path dependencies, and evolutionary dimensions.Journal of Marketing, 60 (4),107-114.

38. Hurley, R. F. \&Hult, G. T. (1998). Innovation, market orientation, and organizational learning: an integration and empirical examination.Journal of marketing, 62 (3), 42-54. 
39. Jaworski, B. J. \& Kohli, A. K. (Julio, 1993). Market orientation: antecedents and consequences. Journal of marketing, 57 (7), 53-70.

40. Jaworsky, B.J., Kohli, A. \&Sahay, A. (Enero, 2000). Market-driven vs driving market.Journal of Academy of Marketing Science, 28 (1), 45-54.

41. Katsikeas, C. S., Piercy, N. F.(1993). Longterm export stimuli and firm characteristics in a European ldc.Journal of international marketing, 1 (3), 23-48.

42. Kirca, A. H., Jayachandran, S. \& Bearden, W. O. (Abril, 2005). Market orientation: a meta- analytic review and assessment of its antecedents and impact on performance. Journal of marketing, 69 (2), 24-41.

43. Kim, W.C. \& Hwang, P. (1992).Global strategy and multinationals entry mode choice.Journal of international business studies, 23 (1),29-53.

44. Knight, G. (2004). International services marketing: review of research.The Journal of Services Marketing. $\mathrm{N}^{\circ}$. 13, pp. 42-45.

45. Kohli, A. K. \&Jaworski, B.J. (Abril, 1990).Market orientation: the construct, research propositions, and managerial implications.Journal of marketing, 54 (4), $1-18$.

46. Kohli, A. K., Jaworski, B. J. \& Kumar, A. (Noviembre, 1993). Markor: a measure of marketing orientation.Journal of marketing research, 30 (11), 467-477.

47. Lado, N. \&Martínez, M.A. (Octubre, 1997). Market orientation and business performance in the Spanish insurance sector.The Genevepaperson Risk and Insurance, 22 (85), 571-590.
48. Lee, T. S. (2005).The effects of business operation mode on market orientation, learning orientation and innovativeness. Industrial marketing and data systems, 105 (3), 325-48.

49. Lehmann, D. R., Gupta, S. \& Steckel, J.H. (1999). Marketing research. New York: Addison-Wesley.

50. Llonch, J. (1993).Orientación al mercado y competitividad de la empresa, Barcelona: Edición Gestión.

51. Llonch, J. y López, P. (2004).La relación entre la orientación al mercado y los resultados y el efecto moderador de la estrategia genérica en dicha relación. Cuadernos de economía y dirección de la empresa, 21, 65-88.

52. Luque, T. (2000).Técnicas de análisis de datos en investigación de mercados. Madrid: Pirámide.

53. Maltz, E. \&Kohli, A. (Febrero, 1996). Market intelligence dissemination across functional boundaries.Journal of marketing research, 3 (2),47-61.

54. Mandowki, E. (1978). La artesanía colombiana.Bogotá: Artesanías de Colombia.

55. Martínez, A.I. (2004). Factores de competitividad en el proceso de internacionalización de las empresas del sector del granito. XII Encuentro de Profesores Universitarios de Marketing, Santiago de Compostela, septiembre, pp. 489- 504.

56. Matsuno, K. \&Mentzer, J. T. (Octubre, 2000). The effects of strategy type on the market orientation-performance relationship. Journal of marketing, 64 (10),1-16. 
57. Matsuno, K., Mentzer, J. T. \&Rentz, J. O. (2000).A refinement and validation of the markor scale.Journal of the academy of marketing science, 28 (4),527-539.

58. Mazaira, A., Dopico, A. \& González, E. (2005).Incidencia del grado de orientación al mercado de las organizaciones empresariales en el desarrollo de las capacidades estratégicas de marketing. Revista europea de dirección y economía de la empresa, 14 (3), 181-208.

59. Mejía, D, I. (2004). La artesanía de México. México: Conacyt.

60. Nahm, V, K. (2005), The impact of organizational structure on time-based manufacturing and performance.Journal of Operations Management. $\mathrm{N}^{\circ} .21$, pp. 281306.

61. Narver, J. C. \& Slater, S. F. (Octubre, 1990). The effect of a market orientation on business profitability.Journal of marketing, 54 (10), 20-35.

62. Narver, J.C., Slater, S.F. \&Tietje, B. (1998).Creating a market orientation. Journal of market focused management, 2 (3), 241-255.

63. Nunnaly, J. (1979). Psychometric Theory. Nueva York: McGraw-Hill.

64. Noble, C. H., Sinha, R. K. \&Kumar, A. (2002). Market orientation and alternative strategic orientations: a longitudinal assessment of performance implications. Journal of marketing, 66, (4), 25-39.

65. Ocampo, J. (1983).Historia del pueblo boyacense.Tunja: Ediciones Instituto de Cultura y Bellas Artes de Boyacá.

66. Oregan, G, S. (2006).Fast tracking innovation in manufacturing SMEs. International Journal of Globalization and Small Business. Vol. 1, N . 4,pp. 407-420.

67. Pabón, L.J. (2008). Planteamiento de una escala preliminar basada en los modelos de orientación al mercado y su validación conceptual en la industria de alimentos en Colombia. Bogotá: Universidad Nacional.

68. Quiñónez, A. C. (2003). Reflexiones en torno a la artesanía y el diseño en Colombia. Bogotá: Universidad Javeriana.

69. Reicheeld, F. (Marzo-abril,1993).Loyaltybased management.Harvard Business Review, 71 (2), 64-73

70. Santos, M. L., Vázquez, R. \& Álvarez, L.I. (2002).La orientación al mercado como fuente de ventajas competitivas: antecedentes asociados al equipo directivo y a la estructura organizativa en las empresas industriales.Revistaasturiana de economía, 25,215-244.

71. Selnes, F., Jaworski, B. J. \& Kohli, A. K. (1996). Market orientation in United States and Scandinavian companies: a crosscultural study.Scandinavian journal of management, 12 (2),139-157.

72. Singh, G. \&Ranchod, F. (2004).Market orientation and customer satisfaction: evidence from British machine tool industry.Industrial Marketing Management, 33, 135-144.

73. Shoham, A., Rose, G. \&Kropp, F. (2005).Market orientation and performance: a meta- analysis.Marketing intelligence \& planning, 23 (5), 435-454.

74. Slater, S. F. \&Narver, J.C.(Enero, 1994). ¿Does competitive environment moderate the market orientation performance 
relationship?Journal of marketing, 58 (1), 46-55.

75. Slater, S.F. \&Narver, J. C. (Julio, 1995). Market orientation and the learning organization.Journal of marketing, 59(7), 63-74.

76. Varela, J. A., Calvo, A. \& Benito, L. $\left(1996^{a}\right)$. Antecedentes ambientales, orientación al mercado y crecimiento de la empresa. Una investigación empírica.VIII encuentro de profesores universitarios de marketing.(pp. 301-310). Llevado a cabo en Zaragoza.
77. Varela, J. A. \& Del Río, M. L. (2003). Market orientation behavior: an empirical investigation using markor.Marketing intelligence \& planning, 21 (1), 6-15.

78. Vásquez, R., Santos, M. L. \&Álvarez, L. I. (2001).Market orientation, innovation and competitive strategies in industrial firms.Journal of strategic marketing, 9, 6990.

79. Venkatraman, N. \&Vasudevan, R. (1986). Measurement of business performance in strategy research: a comparison of approaches.Academy of Management Review, 11 (4),801-814. 